\title{
Tensor Products of the Gassner Representation of The Pure Braid Group ${ }^{\dagger}$
}

\author{
Mohammad N. Abdulrahim*
}

Department of Mathematics, Beirut Arab University, P.O. Box 11-5020, Beirut, Lebanon

\begin{abstract}
The reduced Gassner representation is a multi-parameter representation of $P_{n}$, the pure braid group on $\mathrm{n}$ strings. Specializing the parameters $t_{1}, t_{2}, \ldots, t_{n}$ to nonzero complex numbers $x_{1}, x_{2}, \ldots, x_{n}$ gives a representation $G_{n}\left(x_{1}, \ldots, x_{n}\right): P_{n}$ $\rightarrow G L\left(\mathbb{C}^{n-1}\right)$ which is irreducible if and only if $x_{1} \ldots x_{n} \neq 1$.We find a sufficient condition that guarantees that the tensor product of an irreducible $G_{n}\left(x_{1}, \ldots, x_{n}\right)$ with an irreducible $G_{n}\left(y_{1}, \ldots, y_{n}\right)$ is irreducible. We fall short of finding a necessary and sufficient condition for irreducibility of the tensor product. Our work is a continuation of a previous one regarding the tensor product of complex specializations of the Burau representation of the braid group.
\end{abstract}

Keywords: Braid group, Pure braid group, Gassner representation.

\section{INTRODUCTION}

The pure braid group, $P_{n}$, is a normal subgroup of the braid group, $B_{n}$, on $n$ strings. It has a lot of linear representations. One of them is the Gassner representation which comes from the embedding $P_{n} \rightarrow \operatorname{Aut}\left(F_{n}\right)$, by means of Magnus representation [1, p.119]. According to Artin, the automorphism corresponding to the braid generator $\sigma_{i}$ takes $x_{i}$ to $x_{i} x_{i+1} x_{i}^{-1}, x_{i+1}$ to $x_{i}$, and fixes all other free generators. Applying this standard Artin representation to the generator of the pure braid group, we get a representation of the pure braid group by automorphisms. The Gassner representation is obtained from the Artin representation via the free differential calculus, as is explained in chapter 3 of Birman's book [1]. Such a representation has a composition factor, the reduced Gassner representation $G_{n}\left(t_{1}, \ldots, t_{n}\right): P_{n} \rightarrow$ $G L_{n-1}\left(\mathbb{C}\left[t_{1}^{ \pm 1}, \ldots, t_{n}^{ \pm 1}\right]\right)$, where $t_{1}, \ldots, t_{n}$ are indeterminates. Specializing $t_{1}, \ldots, t_{n}$ to nonzero complex numbers $x_{1}, \ldots, x_{n}$ defines a representation $G_{n}\left(x_{1}, \ldots, x_{n}\right): P_{n} \rightarrow G L_{n-1}(\mathbb{C})=\operatorname{GL}\left(\mathbb{C}^{n-1}\right)$ which is irreducible if and only if $x_{1} \ldots x_{n} \neq 1$ [2]. For some $j$ $\in\{1, \ldots, n\}$, we consider a free normal subgroup of rank $n-1$, namely, $U_{j}$ and consider the complex specialization of the reduced Gassner representation restricted to this free normal subgroup.

In [3], we considered the tensor product of complex specializations of the Burau representation of the braid group and found a necessary and sufficient condition that guarantees irreducibility. Here, we consider the tensor product of the following irreducible representation restricted to a free normal subgroup of the pure braid group, namely, $U_{j}$ :

$$
G_{n}\left(x_{1}, \ldots, x_{n}\right) \otimes G_{n}\left(y_{1}, \ldots, y_{n}\right): U_{j} \rightarrow G L\left(\mathbb{C}^{n-1} \otimes \mathbb{C}^{n-1}\right) .
$$

It is a known fact that tensor products of the classical irreducible representations of the pure braid groups are " generically" irreducible, as they can be obtained by a mono

*Address correspondence to this author at the Department of Mathematics, Beirut Arab University, P.O. Box 11-5020, Beirut, Lebanon; E-mail: mna@bau.edu.lb

${ }^{\dagger}$ Mathematics Subject Classification. Primary: 20F36. dromy construction. For more details, see [4]. As a consequence, the question left to be answered is: For which values of the parameters is the representation above irreducible?

Our main result, Theorem 1 , is that for $n \geq 3$ and $x_{1}, \ldots, x_{n}, y_{1}, \ldots, y_{n} \in \mathbb{C}-\{0,1\}$, the representation above is irreducible under the following condition: for some $i$ and $j \in$ $\{1, \ldots, n\}, i<j, x_{i} x_{j} \neq y_{i} y_{j}$ and $\beta_{a} x_{j} \gamma_{a} y_{j} \neq 1$, for every $\alpha \in\{1, \ldots, n$ $-1\}$.Here $\beta_{\alpha}=x_{\alpha}, \gamma_{\alpha}=y_{\alpha}$ for $\alpha<j$ and $\beta_{\alpha}=x_{\alpha+1}, \gamma_{\alpha}=y_{\alpha+1}$ for $\alpha$ $\geq j$. However, we fail to resolve the question whether or not the tensor product obtained is reducible under each of the following conditions specified because there is no general principle to imply reducibility. In other words, we will only find a sufficient condition for irreducibility.

The idea of the proof is similar to that in [3]. We consider a free normal subgroup of the pure braid group of rank $n-1$ denoted by $U_{j}$ where $1 \leq j \leq n$. Let $\mathbb{C}\left[U_{j}\right]$ be the group algebra of $U_{j}$ over $\mathbb{C}$, and let Abe the augmentation ideal of $\mathbb{C}\left[U_{j}\right]$. If $M$ is any $U_{j}$-module, then $\mathcal{A} M$ is a $U_{j}$-submodule of $M$. We first show (Lemma 2(b)) that if $\mathbb{C}^{n-1}$ is made into a $U_{j}$-module via $G_{n}\left(x_{1}, \ldots, x_{n}\right): U_{j} \rightarrow G L\left(\mathbb{C}^{n-1}\right)$,then $\mathcal{A} \mathbb{C}^{n-1}$ is its unique minimal nonzero $U_{j}$-submodule. Of course $\mathcal{A} \mathbb{C}^{n-1}=$ $\mathbb{C}^{n-1}$ when $G_{n}\left(x_{1}, \ldots, x_{n}\right)$ is irreducible.

Now let $x_{1}, \ldots, x_{n}, y_{1}, \ldots, y_{n} \in \mathbb{C}-\{0\}$, so that $G_{n}\left(x_{1}, \ldots, x_{n}\right) \otimes$ $G_{n}\left(y_{1}, \ldots, y_{n}\right)$ defines a diagonal action of $U_{j}$ on $\mathbb{C}^{n-1} \otimes \mathbb{C}^{n-1}$. The main technical result is Proposition 1, which gives a sufficient condition for $\mathcal{A} \mathbb{C}^{\mathrm{n}-1} \otimes \mathcal{A} \mathbb{C}^{n-1}$ to be the unique minimal nonzero $U_{j}$-submodule of $\mathbb{C}^{n-1} \otimes \mathbb{C}^{n-1}$. This implies the irreducibility of the tensor product above.

\section{DEFINITIONS AND NOTATION}

Notation 1. The pure braid group, $P_{n}$, is defined as the kernel of the homomorphism $B_{n} \rightarrow S_{n}$, defined by $\sigma_{i} \rightarrow(i, i+$ $1), 1 \leq i \leq n-1$. It has the following generators:

$$
A_{i, j}=\sigma_{j-1} \sigma_{j-2} \ldots \sigma_{i+1} \sigma_{i}^{2} \sigma_{i+1}^{-1} \ldots \sigma_{j-2}^{-1} \sigma_{j-1}^{-1}, 1 \leq i<j \leq n
$$


We will construct for each $j=1, \ldots, n$ a free normal subgroup of rank $n-1$, namely, $U_{j}$. Let $U_{j}$ be the subgroup generated by the elements

$$
A_{1, j}, A_{2, j}, \ldots, A_{j-1, j}, A_{j, j+1}, \ldots, A_{j, n},
$$

where $A_{i, j}$ are those generators of $P_{n}$ that become trivial after the deletion of the $j$-th strand. For a fixed value $j$, the image of $A_{i j}$ under the reduced Gassner representation is denoted by $\tau_{\mathrm{i}}$, where $\tau_{i}=\mathrm{I}-P_{i} Q_{i}$. In other words, the generators of $U_{\mathrm{j}}$ are $A_{i, j}$ where $A_{i, j}=A_{j, i}$ whenever $i>j$ and $j=1,2, \ldots, n$. It is known that $U_{j}$ generates a free subgroup of $P_{n}$ which is isomorphic to the subgroup $\mathrm{U}_{\mathrm{n}}$ freely generated by $\left\{A_{1, n}, A_{2, n}, \ldots, A_{n-1, n}\right\}$. This is intuitively clear because it is quite arbitrary how we assign indices to the braid "strings".

For a fixed choice of $\mathrm{j}$, we denote the image of the generator of $U_{j}$, namely, $A_{i, j}$, under the reduced Gassner representation by $\tau_{i}$. That is

$$
\tau_{1}=A_{1, j}, \ldots, \tau_{j-1}=A_{j-1, j}, \quad \tau_{j}=A_{j, j+1}, \quad \tau_{j+1}=A_{j, j+2}, \ldots, \tau_{n^{-1}}=A_{j, n},
$$

Definition 1. The reduced Gassner representation restricted to $U_{j}$ is defined as follows: $\tau_{i}=I-P_{i} Q_{i}$ for $1 \leq i \leq n-1$. For $i<j, P_{i}$ is the column vector given by

$$
\begin{gathered}
(1-t_{1}, \ldots, 1-t_{i-1}, \underbrace{I-t_{i} t_{j}}_{i}, t_{j}\left(1-t_{i+1}\right), \ldots, t_{j}\left(1-t_{j-1}\right), \\
\underbrace{t_{j+1}-1}_{j}, t_{j+2}-1, \ldots, t_{n}-1)^{T},
\end{gathered}
$$

and for $i \geq j, P_{i}$ is the column vector given by

$$
\begin{gathered}
(t_{j}\left(t_{1}-1\right), \ldots, t_{j}\left(t_{j-1}-1\right), \underbrace{1-t_{j+1}, \ldots, 1-t_{i}}_{i-j}, \\
\left.1-t_{i+1} t_{j}, t_{j}\left(1-t_{i+2}\right), \ldots, t_{j}\left(1-t_{n}\right)\right)^{T} .
\end{gathered}
$$

Here $T$ is the transpose and $Q_{i}$ is the row vector given by

$$
Q_{i}=(0, \ldots, 0, \underbrace{1}_{i}, 0, \ldots, 0), 1 \leq i \leq n-1 .
$$

The definition of the reduced Gassner representation restricted to a free normal subgroup is the same, up to equivalence, as the definition in [2]. Representations given by pseudo reflections $I-A_{i} B_{i}$ and $I-C_{i} D_{i}$ are equivalent if the inner products $\left(B_{i} A_{j}\right)$ and $\left(D_{i} C_{j}\right)$ are conjugate by a diagonal matrix. For more details, see [5].

We identify $\mathbb{C}^{n-1}$ with $(n-1) \times 1$ column vectors. We let $e_{1}, \ldots, e_{n^{-1}}$ denote the standard basis for $\mathbb{C}^{n-1}$, and we consider matrices to act by left multiplication on column vectors.

Definition 2. If $r=a_{1} e_{1}+\cdots+a_{n-1} e_{n-1} \in \mathbb{C}^{n-1}$, the support of $r$, denoted $\operatorname{supp}(r)$, is the set $\left\{e_{i} \mid a_{i} \neq 0\right\}$. If $s=\Sigma a_{i j}\left(e_{i} \otimes e_{j}\right) \in$ $\mathbb{C}^{n-1} \otimes \mathbb{C}^{n-1}$, the support of $s$, also denoted $\operatorname{supp}(s)$, is the set $\left\{e_{i} \otimes e_{j} \mid a_{i j} \neq 0\right\}$, and $a_{i j}$ is called the coefficient of $e_{i} \otimes e_{j}$ in $s$.

Definition 3. For $t=\left(t_{1}, \ldots, t_{n}\right)$, we define $v_{i}(t)=e_{i}-\tau_{i}(t)\left(e_{i}\right)=$ $\left(I-\tau_{i}(t)\right)\left(e_{i}\right)$. In other words, we have the following:

For $1 \leq i \leq j-1$, we have $v_{i}(t)=$

$$
\begin{gathered}
(1-t_{1}, \ldots, 1-\underbrace{t_{i-1},}_{i} \underbrace{1-t_{i} t_{j}}_{i}, t_{j}\left(1-t_{i+1}\right), \ldots, t_{j}\left(1-t_{j-1}\right), \\
\underbrace{t_{j+1}-1}_{j}, t_{j+2}-1, \ldots, t_{n}-1)^{T} .
\end{gathered}
$$

and for $i \geq j$, we have $v_{i}(t)=$

$$
\begin{gathered}
(t_{j}\left(t_{1}-1\right), \ldots, t_{j}\left(t_{j-1}-1\right), \underbrace{1-t_{j+1}, \ldots, 1-t_{i}}_{i-j}, \\
\left.1-t_{i+1} t_{j}, t_{j}\left(1-t_{i+2}\right), \ldots, t_{j}\left(1-t_{n}\right)\right)^{T} .
\end{gathered}
$$

Let $v_{1}(t), \ldots, v_{n^{-1}}(t)$ be the columns of the matrix $N\left(t_{1}, \ldots, t_{n}\right)$ defined as follows:

$$
N(t)=\left(\begin{array}{ccccccc}
1-t_{1} t_{j} & \cdots & 1-t_{1} & \mid & t_{j}\left(t_{1}-1\right) & \cdots & t_{j}\left(t_{1}-1\right) \\
t_{j}\left(1-t_{2}\right) & \cdots & 1-t_{2} & \mid & t_{j}\left(t_{2}-1\right) & \cdots & t_{j}\left(t_{2}-1\right) \\
\vdots & & \vdots & & \vdots & \vdots & \vdots \\
t_{j}\left(1-t_{j-1}\right) & \cdots & 1-t_{j-1} t_{j} & \mid & t_{j}\left(t_{j-1}-1\right) & \cdots & t_{j}\left(t_{j-1}-1\right) \\
-- & -- & -- & - & & & \\
t_{j+1}-1 & \cdots & t_{j+1}-1 & & 1-t_{j} t_{j+1} & \cdots & 1-t_{j+1} \\
\vdots & & \vdots & & \vdots & \vdots & \vdots \\
t_{n}-1 & \cdots & t_{n}-1 & t_{j}\left(1-t_{n}\right) & \cdots & 1-t_{n} t_{j}
\end{array}\right)
$$

For $x_{1}, \ldots, x_{n} \in \mathbb{C}-\{0\}$ and $\mathrm{x}=\left(x_{1}, \ldots, x_{n}\right)$, the representation $U_{j}$ $\rightarrow G L_{n-1}(\mathbb{C})=G L\left(\mathbb{C}^{n-1}\right)$ obtained by specializing $t_{i} \rightarrow x_{i}$ is denoted by $\mathrm{G}_{\mathrm{n}}(\mathrm{x})$ and the representation $\mathrm{G}_{\mathrm{n}}(\mathrm{y})$ is defined in the same manner by specializing $t_{i} \rightarrow y_{i}$ and having $y=$ $\left(y_{1}, \ldots, y_{n}\right)$. Also $v_{i}(x), v_{i}(y)$ are defined analogously.

\section{PRELIMINARIES}

Lemma 1 records for future use the action of $\tau_{1}(t), \ldots$, $\tau_{n-1}(t), v_{1}(t), \ldots, v_{n-1}(t)$, and is proved by direct computation.

Lemma 1. For $t=\left(t_{1}, \ldots, t_{n}\right)$, we have

$$
\begin{aligned}
& \tau_{i}(t)\left(v_{i}(t)\right)=t_{i} t_{j} v_{i}(t) \\
& \tau_{i}(t)\left(v_{i}(t)\right)=t_{i+1} t_{j} v_{i}(t) \\
& \tau_{i}(t)\left(v_{k}(t)\right)=v_{k}(t)+\left(t_{i+1}-1\right) v_{i}(t) \\
& \tau_{i}(t)\left(v_{k}(t)\right)=v_{k}(t)+\left(t_{i}-1\right) v_{i}(t) \\
& \tau_{i}(t)\left(v_{k}(t)\right)=v_{k}(t)+t_{j}\left(1-t_{i}\right) v_{i}(t) \\
& \tau_{i}(t)\left(v_{k}(t)\right)=v_{k}(t)+\left(1-t_{i+1}\right) v_{i}(t) \\
& \tau_{i}(t)\left(v_{k}(t)\right)=v_{k}(t)+t_{j}\left(t_{i}-1\right) v_{i}(t) \\
& \text { for } 1 \leq i \leq j-1 \text {, } \\
& \text { for } 1 \leq \mathrm{j} \leq i \text {, } \\
& \text { for } j \leq i<k \text {, } \\
& \text { for } i<k<j \text {, } \\
& \text { for } i<j \leq k \text {, } \\
& \tau_{i}(t)\left(v_{k}(t)\right)=v_{k}(t)+t_{j}\left(t_{i+1}-1\right) v_{i}(t) \\
& \text { for } k<j \leq i \text {, } \\
& \text { for } k<i<j \text {, } \\
& \text { for } j \leq k<\mathrm{i} \text {. }
\end{aligned}
$$

Note that Lemma 1 remains true for any specialization $t_{i}$ $\rightarrow x_{i}$,where $x_{i} \in \mathbb{C}^{*}$. For simplicity, we denote $\left(x_{1}, \ldots, x_{n}\right)$ by the vector $x$.

Lemma 2. Having $U_{j}$ a free normal subgroup of the pure braid group, we let $G_{n}(x): U_{j} \rightarrow G L\left(\mathbb{C}^{n-1}\right)$ be a specialization of the Gassner representation restricted to $U_{j}$ making $\mathbb{C}^{n-1}$ into a $U_{j}$-module, where $n \geq 3$. Then

(a) Let $\mathcal{A}$ be the kernel of the homomorphism $\mathbb{C}\left[U_{j}\right] \rightarrow \mathbb{C}$ induced by $\tau_{i} \rightarrow 1$ (the augmentation ideal). Then $\mathcal{A} \mathbb{C}^{\mathrm{n}^{-1}}$ is equal to the $\mathbb{C}$-vector space spanned by $v_{1}(x), \ldots, v_{n^{-1}}(x)$.

(b) If $M$ is a nonzero $U_{j}$-submodule of $\mathbb{C}^{n-1}$, then $\mathcal{A} \mathbb{C}^{n-1} \subseteq$ $M$. Hence $\mathcal{A} \mathbb{C}^{n-1}$ is the unique minimal nonzero $U_{j^{-}}$ submodule of $\mathbb{C}^{n-1}$.

If $p(x)=\left(x_{j}-1\right)^{n-2}\left(x_{1} x_{2} \ldots x_{n}-1\right) \neq 0$, then $\mathcal{A} \mathbb{C}^{n^{-1}}=$ $\mathbb{C}^{n^{-1}}$, and $G_{\mathrm{n}}\left(x_{1}, x_{2}, \ldots, x_{n}\right)$ is irreducible.

Proof. The proof is similar to that in [3, p.107]. Here, we will take the free normal subgroup, $U_{j}$, of rank $n-1$ instead of the braid group, $B_{n}$. Notice that, in the proof of (b), we 
need the fact that if $v_{j} \in M$ for some $j$ then all $v_{i} \in M$. This is due to Lemma 1. As for (c), the determinant of $N\left(x_{1}, \ldots, x_{n}\right)$ is $p(x)=\left(x_{j}-1\right)^{n-2}\left(x_{1} x_{2} \ldots x_{n}-1\right)$, so if $p(x) \neq 0$ then $v_{1}(x), \ldots v_{n-1}(x)$ is a basis for $\mathbb{C}^{n-1}$ and $\mathcal{A} \mathbb{C}^{n-1}=\mathbb{C}^{n-1}$. For more details, see [2].

\section{PROOF OF THE MAIN THEOREM}

Proposition 1. Suppose that $x=\left(x_{1}, \ldots, x_{n}\right)$ and $y=\left(y_{1}, \ldots, y_{n}\right) \in$ $\mathbb{C}^{n}$, where $x_{s}, y_{s} \in \mathbb{C}-\{0,1\}$ for $1 \leq s \leq n$, and for some $i$ and $j(i<j)$, we have $x_{i} x_{j} \neq y_{i} y_{j}, x_{\alpha} x_{j} y_{a} y_{j} \neq 1$ for every $\alpha \in\{1, \ldots, j-$ $1\}$ and $x_{\alpha+1} x_{j} y_{\alpha+1} y_{j} \neq 1$ for every $\alpha \in\{j, \ldots, n-1\}$. Let $M$ be a nonzero $U_{j}$-submodule of $\mathbb{C}^{n-1} \otimes \mathbb{C}^{n^{-1}}$ under the action of $G_{n}(x) \otimes G_{n}(y): U_{j} \rightarrow G L\left(\mathbb{C}^{n-1} \otimes \mathbb{C}^{n-1}\right)$, where $n \geq 3$. Then $M$ contains all $v_{i}(x) \otimes v_{j}(y)$ for $1 \leq i, j \leq n-1$. Thus $M$ contains $\mathcal{A} \mathbb{C}^{n^{-1}} \otimes \mathcal{A} \mathbb{C}^{n^{-1}}$, where the action of $U_{j}$ on the first factor is induced by $G_{n}\left(x_{1}, \ldots, x_{n}\right)$ and the action of $U_{j}$ on the second factor is induced by $G_{n}\left(y_{1}, \ldots, y_{n}\right)$.

Proof. For $1 \leq j \leq n$, we consider the normal free subgroup of rank $n-1$, namely, $U_{j}$, defined as before.

First, we observe that if $e_{u} \otimes e_{v} \in \operatorname{supp}(m)$ for some $m \in$ $M$ then $e_{\alpha} \otimes e_{v} \in \operatorname{supp}\left(\tau_{u}\left(e_{u} \otimes e_{v}\right)\right)$ for every choice of $\alpha=$ $1, \ldots, n-1$ and $v \neq u$. This is clear because of our assumption that none of the parameters $t_{i}$ 's is equal to zero or one.

Claim 1. There exists an $s \in\{1, \ldots, n-1\}$ such that $e_{s} \otimes e_{s} \in$ $\operatorname{supp}(m)$ for some $m \in M$.

Proof of Claim 1.

Case 1: Suppose that there exists an $s$ and $m \in M$ such that $e_{s} \otimes e_{s} \in \operatorname{supp}(m)$, then we are done.

Case 2: Suppose that there exists $(s, t)$ with $1 \leq s, t \leq n-1$ and $s \neq t$ such that

$m=a\left(e_{s} \otimes e_{t}\right)+W$,

where $\mathrm{a} \in \mathbb{C}^{*}$ and $\operatorname{supp}(W)$ does not contain $e_{s} \otimes e_{t}, e_{t} \otimes e_{s}$. We also assume that $\operatorname{supp}(W)$ does not contain $e_{\alpha} \otimes e_{\alpha}$ for any $\alpha$.

Then $\tau_{t}(m)=a\left(e_{s} \otimes e_{t}-v_{\mathrm{t}}\right)+\tau_{t}(W)$, which implies that $e_{s}$ $\otimes e_{s} \in \operatorname{supp}\left(\tau_{t}(m)\right)$ and so we are done .

Case 3: Suppose that for any pair $(s, t)$ and any $m \in M$ such that $e_{s} \otimes e_{t} \in \operatorname{supp}(m)$, we have that $e_{t} \otimes e_{s} \in \operatorname{supp}(m)$ as well. That is, consider $m \in M$ such that

$$
m=a\left(e_{s} \otimes e_{t}\right)+b\left(e_{t} \otimes e_{s}\right)+W, \text { where }
$$

$\operatorname{supp}(W)$ does not contain $e_{s} \otimes e_{t}, e_{t} \otimes e_{s}$ and $e_{\alpha} \otimes e_{\alpha}$ for any $\alpha$. In this case, $W$ is either zero or its elements are of the form $\sum_{k, l}\left(c_{k, l} e_{k} \otimes e_{l}+\mathrm{d}_{l, k} e_{l} \otimes e_{k}\right)$

Here the constants $a, b, c_{k, l}, d_{l, k} \in \mathbb{C}^{*}$.

Applying $\tau_{t}$,we observe that $e_{i} \in \operatorname{supp}\left(\tau_{t}\left(e_{t}\right)\right)$, where $i$ is the integer given by the hypothesis of Proposition 1 . Then

$$
\tau_{t}(m)=a\left(e_{s} \otimes e_{i}\right)+b\left(e_{i} \otimes e_{s}\right)+W, \text { where }
$$

$\operatorname{supp}(W)$ does not contain $e_{s} \otimes e_{i}, e_{i} \otimes e_{s}$, and both of $a, b$ are not zeros. For simplicity, we denote $\tau_{t}(m)$ by $m$.

If $e_{\alpha} \otimes e_{\alpha} \in \operatorname{supp}(W)$ for some $\alpha$, then we are done. If not, we see that $a M+b N=$ coefficient of $e_{s} \otimes e_{s}$ in $\tau_{i}(m)$ and $a M\left(1+y_{i} y_{j}\right)+$ $b N\left(1+x_{i} x_{j}\right)=$ coefficient of $e_{s} \otimes e_{s}$ in $\tau_{i}^{2}(m)$.

The values of $M$ and $N$ are not zeros and can be obtained directly from Definition 3. The determinant

$$
\operatorname{det}\left(\begin{array}{cc}
M & N \\
M\left(1+y_{i} y_{j}\right) & N\left(1+x_{i} x_{j}\right)
\end{array}\right)=M N\left(x_{i} x_{j}-y_{i} y_{j}\right)
$$

is nonzero, since $x_{i} x_{j}-y_{i} y_{j} \neq 0$ by hypothesis. Then one of $\tau_{i}(m),\left(\tau_{i}\right)^{2}(m)$ has $e_{s} \otimes e_{s}$ in its support.

Claim 2. Suppose that $e_{\alpha} \otimes e_{\alpha} \in \operatorname{supp}(m)$ for some $m \in M$. Then $v_{\alpha}(x) \otimes v_{\alpha}(y) \in M$ if $x_{\alpha} x_{j} y_{\alpha} y_{j} \neq 1$ for $\alpha=1, \ldots, j-1$ and $x_{\alpha+1} x_{j} y_{\alpha+1} y_{j} \neq 1$ for $\alpha=j, \ldots, n-1$.

Proof of Claim 2. A calculation shows that

$$
\begin{gathered}
\left(\tau_{\alpha}-1\right)\left(\tau_{\alpha}-\gamma_{\alpha} y_{j}\right)\left(\tau_{\alpha}-\beta_{\alpha} x_{j}\right)\left(\mathrm{e}_{\alpha} \otimes e_{\alpha}\right)=\gamma_{\alpha} \beta_{\alpha} x_{j} y_{j}\left(\beta_{\alpha} x_{j} \gamma_{\alpha} y_{j}-1\right) \\
\left(v_{\alpha}(x) \otimes v_{\alpha}(y)\right)
\end{gathered}
$$

and

$\left(\tau_{\alpha}-1\right)\left(\tau_{\alpha}-\gamma_{\alpha} y_{j}\right)\left(\tau_{\alpha}-\beta_{\alpha} x_{j}\right)\left(e_{u} \otimes e_{v}\right)=0$ if $(u, v) \neq(\alpha, \alpha)$.

Here we have

$$
\beta_{\alpha}=x_{\alpha}, \gamma_{\alpha}=y_{\alpha} \text { for } \alpha=1, \ldots, j-1
$$

and

$$
\beta_{\alpha}=x_{\alpha+1}, \gamma_{\alpha}=y_{\alpha+1} \text { for } \alpha=j, \ldots, n-1 .
$$

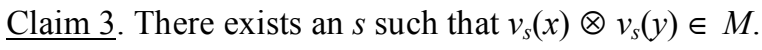

Proof of Claim 3. There exists, by claim1, an element $e_{s}$ $\otimes e_{s} \in \operatorname{supp}(m)$ for some $m \in M$. By Proposition 1 , we have $\beta_{\alpha} x_{j} \gamma_{\alpha} y_{\mathrm{j}} \neq 1$ for all $\alpha=1, \ldots, n-1$. It follows, by claim 2 , that $v_{s} \otimes v_{s} \in M$.

Claim 4. For $\alpha=1, \ldots, n-1$, we have that $v_{\alpha}(x) \otimes v_{\alpha}(y) \in M$.

Proof of Claim 4. We have, by claim 3, an integer $s$ such that $v_{s}(x) \otimes v_{s}(y) \in M$.

It follows that $\left(\sum_{k=1}^{n-1} A_{i} e_{i}\right) \otimes\left(\sum_{k=1}^{n-1} B_{i} e_{i}\right) \in M$, which implies that $e_{\alpha}(x) \otimes e_{\alpha}(y) \in \operatorname{supp}(m)$ for some $m \in M$. Since $\beta_{\alpha} x_{j} \gamma_{\alpha} y_{j}$ $\neq 1$, it follows, by claim 2 , that for $\alpha=1, \ldots, n-1$, we have

$$
v_{\alpha}(x) \otimes v_{\alpha}(y) \in M .
$$

Here $A_{i}$ and $B_{i}$ are non zero numbers determined in Definition 3.

Claim 5. For the value $i$ given by Proposition 1, we have that $v_{i} \otimes v_{\alpha} \in M$ and $v_{\alpha} \otimes v_{i} \in M$ for all $\alpha=1, \ldots, n-1$.

Proof of Claim 5. Given an integer $\alpha=1, \ldots, \mathrm{n}-1$. If $\alpha=i$ then we are done by claim 4 . Assume then that $\alpha \neq i$. Since $v_{\alpha} \otimes v_{\alpha} \in M$ for every $\alpha$, it follows that $\tau_{i}\left(v_{\alpha} \otimes v_{\alpha}\right) \in M$. It follows that

$a v_{i} \otimes v_{\alpha}+b v_{\alpha} \otimes v_{i} \in M$,

Applying $\tau_{i}$ again, we obtain that

$a x_{i} x_{j} v_{i} \otimes v_{\alpha}+b y_{i} y_{j} v_{\alpha} \otimes v_{i} \in M$.

(1) and (2) imply that

$$
b\left(x_{i} x_{j}-y_{i} y_{j}\right) v_{\alpha} \otimes v_{i} \in M .
$$

By our hypothesis, we get that 


$$
v_{\alpha} \otimes v_{i} \in M \text { and } v_{i} \otimes v_{\alpha} \in M .
$$

Claim 6. $v_{\alpha} \otimes v_{\beta} \in M$ for all $\alpha, \beta \in\{1,2, \ldots, n-1\}$.

Proof of Claim 6. Fix the value of $\beta$. Let $\alpha \in\{1, \ldots, n-$ $1\}$ and $\alpha \neq \beta$. Then by claim 5, we get that $v_{i} \otimes v_{\alpha}, v_{i} \otimes v_{\beta} \in M$ and so

$$
\tau_{\alpha}\left(v_{i} \otimes v_{\beta}\right) \in M .
$$

This implies that

$$
v_{\alpha} \otimes v_{\beta} \in M .
$$

Having done this for every value of $\alpha$, and consequently $\beta$, this would complete the proof.

Let $x=\left(x_{1}, \ldots, x_{n}\right)$ and $y=\left(y_{1}, \ldots, y_{n}\right) \in \mathbb{C}^{n}$, where $x_{s}, y_{s} \in \mathbb{C}$ $-\{0,1\}, 1 \leq s \leq n$.

Theorem 1. Let $G_{n}(x)$ and $G_{n}(y)$ be the representations : $U_{j} \rightarrow$ $G L\left(\mathbb{C}^{n-1}\right)$ that denote the specializations of the reduced Gassner representation restricted to the free normal subgroup of the pure braid group, namely, $P_{n}$, where $x_{1}, \ldots, x_{n}, y_{1}, \ldots, y_{n} \in \mathbb{C}-\{0,1\}$. Let $p(t)=\left(t_{j}-1\right)^{n-2}\left(t_{1} t_{2} \ldots t_{n}-1\right)$. For $n \geq 3$, consider the tensor product of irreducible representations $G_{n}(x) \otimes G_{n}(y): U_{j} \rightarrow G L\left(\mathbb{C}^{n-1} \otimes \mathbb{C}^{n-1}\right)$, where $p(x) \neq 0$ and $p(y) \neq 0$.

If $x_{i} x_{j} \neq y_{i} y_{j}$ for some $i<j, x_{\alpha} x_{j} y_{\alpha} y_{j} \neq 1$ for $\alpha=1, \ldots, j-1$ and $x_{\alpha+1} x_{j} y_{\alpha+1} y_{j} \neq 1$ for $\alpha=j+1, \ldots, n-1$ then the above representation is irreducible.

Proof. The proof is the same as in [3]. By Proposition 1, $\mathcal{A} \mathbb{C}^{n-1} \otimes \mathcal{A} \mathbb{C}^{n-1}$ is the unique minimal nonzero $U_{j}$-submodule of $\mathbb{C}^{n-1} \otimes \mathbb{C}^{n-1}$. In particular, it is an irreducible $U_{j}$-module. By Lemma 2 and the fact that $p(x) \neq 0, p(y) \neq 0$, the left factor $\mathcal{A} \mathbb{C}^{n-1}$ corresponds to the representation $G_{n}(x)$ and the right factor $\mathcal{A} \mathbb{C}^{n-1}$ corresponds to the representation $G_{n}(y)$.

Since irreducibility on a subgroup implies irreducibility on the group itself, it follows that Theorem 1 is also true for the tensor products of specializations of the Gassner representation of the pure braid group with $n$ strings. Therefore, we get the following corollary.

Corollary 1. Suppose that for $x_{1}, \ldots, x_{n}, y_{1}, \ldots, y_{n} \in \mathbb{C}-\{0,1\}$ and for some $i, j$, we have $x_{i} x_{j} \neq y_{i} y_{j}$ for $1 \leq i<j \leq n$ and $\beta_{\alpha} x_{j} \gamma_{\alpha} y_{j} \neq 1$ for all $\alpha=1, \ldots, n-1$. Here $\beta_{\alpha}, \gamma_{\alpha}$ are defined in claim 2 in the proof of Proposition 1. Then the following representation is irreducible:

$$
G_{n}(x) \otimes G_{n}(y): P_{n} \rightarrow \mathrm{GL}\left(\mathbb{C}^{n-1} \otimes \mathbb{C}^{n-1}\right) \text {, where } p(x) \neq 0, p(y) \neq 0 .
$$

Our work is an extension of a previous one, where we have proved in [3] that the representation obtained, by tensoring irreducible complex specializations of the Burau representation, namely, $\beta_{n}(x)$ or $\hat{\beta}_{n}(x)$ and $\beta_{n}(y)$ or $\hat{\beta}_{n}(y)$ is irreducible if and only if $x \neq y$.

\section{REMARKS}

(1) In our work, We have found a sufficient condition for irreducibility of the representation obtained by tensoring complex specializations of the reduced Gassner representation restricted to a free normal subgroup, but we fall short of finding a necessary and sufficient condition for irreducibility of the tensor product. In the proof of our main theorem, we needed to find a pair $(i, j)$ such that $x_{i} x_{j} \neq y_{i} y_{j}$; whereas for each $\alpha \neq j$, we needed the conditions $\beta_{\alpha} x_{j} \gamma_{\alpha} y_{j} \neq 1$ to show that $v_{\alpha} \otimes v_{\alpha} \in M$. (See claim 2 in the proof of Proposition 1). Based on the proof of Proposition 1, if we could find pure braid elements in $U_{j}$, namely, $\gamma_{m}$, such that $\gamma_{m}\left(v_{m}\right)=a v_{m+1}, m=1, \ldots, n-1$ then we could show, by induction, that if $v_{m} \otimes v_{m} \in M$ then $v_{m^{+1}} \otimes v_{m^{+1}} \in M$ for all values of $\mathrm{m}$. In that case, the sufficient condition for irreducibility in Proposition 1 would be simpler and replaced by the following condition:

$$
x_{i} x_{j} \neq y_{i} y_{j} \text { and } \beta_{\alpha} x_{j} \gamma_{\alpha} y_{j} \neq 1 \text { for some values of } i, j, \alpha
$$

Some further work is needed to investigate whether or not there are pure braids in $U_{j}$, namely, $\gamma_{m}$ such that $\gamma_{m}\left(v_{m}\right)=$ $a v_{m^{+1}}, m=1, \ldots, n-1$.

(2) In [2], it was proved that if $p(x)=\left(x_{j}-1\right)^{n-2}\left(x_{1} \ldots x_{n}-\right.$ $1) \neq 0$ then the representation $G_{n}\left(x_{1}, \ldots, x_{n}\right): U_{j} \rightarrow G L\left(\mathbb{C}^{n-1}\right)$ is irreducible. In a future work, we will attempt to describe the composition factors of $G_{n}\left(x_{1}, \ldots, x_{n}\right)$ when it is reducible, that is when $p(x)=0$. In other words, it will be useful to answer the following question:

If $G_{n}(x): P_{n} \rightarrow G L\left(\mathbb{C}^{n-1}\right)$ is irreducible, when and how uniquely can it be extended to an irreducible representation $G_{n}\left(x_{1}, \ldots, x_{n}\right): P_{n+1} \rightarrow G L\left(\mathbb{C}^{n-1}\right)$ ? This question was answered in the case of the braid group [5, p.284]. If we succeed to answer the question above regarding the pure braid group then the statements in Theorem 1 and Corollary 1 will also hold true for the tensor products of irreducible $G_{n}\left(x_{1}, \ldots, x_{n}\right)$ or $G_{n-1}\left(x_{1}, \ldots, x_{n}\right)$ with an irreducible $G_{n}\left(y_{1}, \ldots, y_{n}\right)$ or $G_{n-1}\left(y_{1}, \ldots, y_{n}\right)$.

\section{REFERENCES}

[1] Birman JS. Braids, links and mapping class groups. N.J: Princeton University Press, 1975.

[2] Abdulrahim M. Complex specializations of the reduced Gassner representation of the pure braid group. Proc Amer Soc 1997; 125(6): 1617-24.

[3] Abdulrahim M, Formanek E. Tensor products of specializations of the burau representation. J Pure Appl Algebra 2005; 203(1-3): 104-12.

[4] Marin I. Irreductibilite' generique des produits tensoriels de monodromies'. Bull Soc Math France 2004; 132(2): 201-32.

[5] Formanek E. Braid group representations of low degree. Proc Lond Math Soc 1996; 73: 279-322. 